\title{
ĐÁNH GIÁ DỊ THƯờnG TRỌNG LỰC CỦA MÔ HÌNH TRỌNG TRƯờNG TOÀN CẦU BẰnG CÁC DŨ LIỆU DI THƯỜNG TRỌNG LỰC ĐO TRỰC TIẾP TRÊN BIỄN VIỆT NAM
}

\author{
LÊ MINH(1), NGUY ̃̂̃N TUẤN ANH(2) \\ (I) Hội Trắc địa-Bản đồ và Viễn thám Việt Nam \\ ${ }^{(2)}$ Cục Đo đạc bản đồ và Thông tin địa lý Việt Nam
}

\section{Tóm tắt:}

Sủ dụng các dũ liệu trọng lực vệ tinh là một trong nhũng giải pháp quan trọng và thiết thực trong việc xây dưng CSDL trọng lục thống nhất phủ kín toàn bộ lãnh thổ và lãnh hải Việt Nam. Nhiệm vu được đặt ra là nghiên cứu xác định mô hình trọng truoòng toàn cầu có độ chính xác và độ tin cậy tốt nhất ở Việt Nam trên co sở đó kết hợp với các dư liệu đo trong lục trực tiếp trên biển để xây dựng CSDL lấp kín toàn bộ vùng biển thuộc quyền quản lý của Việt Nam. Tác giả đã nghiên cứu đánh giá dũ liệu trọng lục tù 4 mô hình trọng trường có độ phân giải cao là Mô hình EGM 2008 có n=2160, Mô hình GECO độ phân giải 2190, Mô hình EIGEN $6 C 4$ độ phân giải 2190 và Mô hình SGG-UGM1 có $n=2159$, với các dũ liệu đo trong lục trục tiếp độc lập trên biển Việt Nam. Việc đánh giá đurợc tiến hành trên khu vưc vịnh Bắc bộ và khu vực thuộc biển Hà Tiên-Phú Quốc. Kết quả cho thấy mô hình GECO đạt độ chính xác tốt nhất với 4,3 mGal khu vục vịnh Bắc bộ và $3 m$ Gal khu vục Hà TiênPhú Quốc. Với kết quả trên mô hình trọng truờng toàn cầu GECO sẽ được nghiên cứu sủ dụng kết hợp với dũ liệu đo trọng lục trục tiếp để xây dựng CSDL trọng lục phủ kín toàn bộ vùng biển thuộc quyền quản lý khai thác của Việt Nam.

\section{Mở đầu}

CSDL trường trọng lực là một dữ liệu điều tra cơ bản quan trọng không những của mỗi Quốc gia mà cả trên phạm vi toàn cầu. Trong nhiều năm qua, các cơ quan, tổ chức quốc tế trên thế giới không ngừng nghiên cứu, thu thập dữ liệu mặt đất và phóng nhiều thế hệ vệ tinh với các phương pháp, giải pháp quan trắc, thu thập dữ liệu khác nhau nhằm xây dựng được một CSDL trường trọng lực toàn cầu với độ chính xác và mật độ dữ liệu cao nhất có thể. Giải pháp xây dựng CSDL trường trọng lực cục bộ dựa trên việc sử dụng kết hợp mô hình trọng trường toàn cầu và dữ liệu đo trọng lực trực tiếp đã được nhiều nước trên thế giới sử dụng. Như đã biết, xây dựng trường trọng lực toàn cầu từ quan trắc vệ tinh ngày càng phát triển và có những bước tiến bộ đáng kể. Gần đây có các dự án đo trọng lực vệ tinh được thực hiện đã cho phép không chỉ xác định trường trọng lực trái đất mà còn xác định sự biến thiên của nó theo thời gian. Các dự án đo trọng lực vệ tinh CHAMP (3/2000), GRACE (9/2002- 2009) và GOCE (2009-2013) đã cho phép xác định mô hình trường trọng lực trái đất chỉ từ dữ liệu trọng lực vệ tinh. Tuy vậy, các mô hình trên có độ phân giải thấp chỉ tới bậc 300 , đây là mô hình trọng trường tương đối đồng nhất và độc lập với dữ liệu trọng lực mặt đất. Nhiều mô hình trọng trường toàn cầu đã được xây dựng với độ phân giải ngày càng cao, trên cơ sở kết hợp giữa trọng lực mặt đất và các dữ liệu quan trắc từ vệ tinh. Mô hình trọng trường toàn cầu được xây dựng kết hợp dựa trên các dữ liệu trọng lực đã được lấy trung bình theo các ô chuẩn 30'x30' hoặc lớn hơn từ các dữ liệu trọng lực đo trực tiếp, kết hợp với dữ liệu vệ tinh cho phép xác định được các mô hình trọng trường có độ phân giải cao tiếp cận khá chính xác với trường trọng lực thực toàn cầu ở các bước sóng trung và sóng dài. Các mô hình kết hợp trên có độ phân giải cao tới bậc 2160 như mô hình EGM2008, hoặc tới bậc 2190 như mô hình EIGEN6C4. Tuy nhiên, việc xây dựng các mô hình

Ngày nhận bài: 07/8/2019, ngày chuyển phản biện: 12/8/2019, ngày chấp nhận phản biện: 15/8/2019, ngày chấp nhận đăng: 20/8/2019 
trọng trường trái đất dựa trên các dữ liệu dị thường trọng lực đã được lấy trung bình trên các ô chuẩn đã làm cho mô hình trơn và đồng điệu hơn, các bước sóng ngắn và cực ngắn đã được làm trơn dẫn đến mô hình kém chính xác hơn. Khảo sát đánh giá các mô hình trọng trường trái đất đã được nhiều nước trên thế giới thực hiện cho các mục đích trắc địa, địa vật lý và địa chất. Các nhà nghiên cứu Phần Lan đã đánh giá các mô hình GOCE với các dữ liệu mặt đất cho độ chính xác khoảng 10 mGal (Saari \&Bieker Koivula 2015), các nghiên cứu của M.Sprlak, C.Gerlach và B.R Pettersen ở Nauy cũng cho kết quả tương tự. Các khảo sát ở Canada cho thấy độ chính xác của mô hình EGM2008 đạt $5 \mathrm{mGal}$ cho vùng Biển (Huang et at 2009), một nghiên cứu của $\mathrm{C}$. Hirt, T. Grubec và Featherstone ở Thuỵ Sỹ và Australia năm 2014 cho thấy độ chính xác dị thường khoảng không tự do của mô hình EGM2008 khi so sánh trên 31.519 điểm trọng lưc đo trực tiếp ở vùng núi Thuỵ Sỹ đạt độ chính xác 4,48 mGal có cải chính địa hình RTM và chỉ còn đạt 39,50 mGal khi không cải chính RTM. Tương tự, kiểm tra trên 28.551 điểm trọng lực ở Australia kết quả $3.40 \mathrm{mGal}$ khi chưa cải chính địa hình và $2.68 \mathrm{mGal}$ khi có cải chính RTM. Đáng chú ý về nghiên cứu độ chính xác của các mô hình kết hợp độ phân giải cao ở Iran ở đất liền cho thấy độ lệch chuẩn của dị thường khoảng không tự do của mô hình EIGEN 6C4 là 11,4 mGal, của mô hình GECO là 11,2 mGal và EGM 2008 là 11,0 mGal (I Foroughi, Y Afarasteh -Iran 2017), sai số trên ngoài sai số mô hình trọng trường do còn chưa được hiệu chỉnh số cải chính địa hình nên các sai số còn lớn. Ở Việt Nam trong đề tài nghiên cứu khoa học về xây dựng mô hình Geoid độ chính xác cao đã kiểm tra độ chính xác của mô hình EGM96 trên các điểm trọng lực mặt đất kết quả cho thấy độ lệch ở vùng núi khoảng $20 \mathrm{mGal}-38 \mathrm{mGal}$, vùng đồng bằng khoảng từ 13-18 mGal. Độ lệch lớn trên một phần do chưa được hiệu chính trọng lực mặt đất và do ảnh hưởng của cải chính địa hình chưa được tính cho mô hình trọng trường toàn cầu (Đặng Hùng Võ, Lê Minh 2003). Gần đây đánh giá độ chính xác của các mô hình trong lực toàn cầu với các dữ liệu đo trọng lực biển ở vùng biển Quảng Ninh, Việt Nam, Nguyễn Văn Sáng (2016) đã cho những kết quả sau: EGM96 (13,6 mGal) EGM2008 (4,9mGal), GO_CONS_EGM_DIR_21 (14,7 mGal) và GOCE-DIR4 $(15,7 \mathrm{mGal})$. Độ chính xác của các mô hình được đánh giá ở trên chưa cao chủ yếu do sai số của mô hình trọng trường và các mô hình trọng trường chưa được hiệu chỉnh đầy đủ số cải chính địa hình.

Mục tiêu của nghiên cứu này là khảo sát phân tích đánh giá độ chính xác của các mô hình trọng trường toàn cầu với các dữ liệu đo trực tiếp trên biển ở Việt Nam để tìm ra mô hình có độ chính xác và độ tin cậy tốt nhất. Trên cơ sở đó làm khớp với các dữ liệu đo trực tiếp để hiệu chỉnh và nâng cao độ chính xác mô hình trọng trường toàn cầu đáp ứng các yêu cầu cho việc xây dựng CSDL trường trọng lực phủ trùm toàn bộ vùng biển Việt Nam với độ chính xác $3 \mathrm{mGal}$. Qua khảo sát cho thấy các mô hình có độ phân giải cao được xây dựng kết hợp từ nhiều nguồn dữ liệu khác nhau như mô hình GECO có bậc 2190 có sự kết hợp giữa các dữ liệu mô hình GOCE độ phân giải tới bậc 300 với mô hình EGM2008 độ phân giải tới bậc 2160, hoặc mô hình EIGEN 6C4 cũng có bậc 2190 là sự kết hợp của 4 loại dữ liệu khác nhau như: Dữ liệu mô hình EGM2008, dữ liệu mô hình vệ tinh Goce $(\mathrm{S})$, Dũ liệu vệ tinh Lageos $(\mathrm{L})$, Dữ liệu vệ tinh Grace $(\mathrm{S})$ và một số mô hình khác cho kết quả tốt hơn nhiều so với mô hình trọng lực chỉ sử dụng một nguồn dữ liệu. Việc khảo sát đánh giá các mô hình trọng trường toàn cầu có độ phân giải cao được so sánh phân tích với các các dữ liệu độc lập đo trọng lực trực tiếp, để có kết quả tốt đòi hỏi phải xem xét phân tích các nguồn sai số của các dữ liệu trên một cách kỹ càng từ đó có các đánh giá khách quan và chính xác.

\section{Cơ sở xác định sai số dị thường trọng lực của mô hình trọng trường toàn cầu}

Dị thường trọng lực xác định theo các hệ số của các hàm điều hòa cầu được biểu thị theo công thức sau: 


$$
\Delta \mathrm{g}=\frac{G M}{r^{2}} \sum_{n=2}^{n \max }\left(\frac{R}{r}\right)^{n}(n-1) \sum_{m=0}^{n}\left[\bar{C}_{n, m} \cos m \lambda+\bar{S}_{n, m} \sin m \lambda\right] \overline{P_{n, m}}(\sin \psi)
$$

Trong đó:

$\lambda, \psi$ - Toạ độ cầu của điểm cần tính

R - Bán kinh của Ellipsoid tham chiếu

$\mathrm{GM}$ - Hằng số trọng trường Trái đất

$\mathrm{n}, \mathrm{m}$ - Hạng và bậc của hệ số điều hoà cầu

$\bar{C}_{\mathrm{n}, \mathrm{m}}, \bar{S}_{\mathrm{n} m \mathrm{~m}}-\mathrm{Hệ} \mathrm{số} \mathrm{hàm} \mathrm{điều} \mathrm{hoà} \mathrm{cầu} \mathrm{chuẩn} \mathrm{hoá}$

$\bar{P}(\sin \psi)$ - Hàm Legendre chuẩn hoá

$\bar{P}_{n m}(\sin \psi)$ - là hàm Legendre chuẩn hóa, được tính bằng phương trình (Bernhard Hofmann, Wellenhof Helmut Moritz, 2005):

$$
\begin{aligned}
\bar{P}_{n m}(\sin \psi) & =\left[\frac{(n-m) !(2 n+1) k}{(n+m) !}\right]^{1 / 2} P_{n m}(\sin \psi) \\
P_{n m}(\sin \psi) & =(\cos \psi)^{m} \frac{d^{m}}{d(\sin \psi)^{m}}\left[P_{n}(\sin \psi)\right]
\end{aligned}
$$

Đa thức Legendre $P_{n}(\sin \psi)$ được tính theo:

$$
P_{n}(\sin \psi)=\frac{1}{2^{n} n !} \frac{d^{n}}{d(\sin \psi)^{n}}\left(\sin ^{2} \psi-1\right)^{n}
$$

Ở đây:

$$
\bar{C}_{n m}=\frac{1}{N_{n m}} C_{n m} ; \bar{S}_{n m}=\frac{1}{N_{n m}} S_{n m}
$$

Hay ở dạng:

$$
\left|\begin{array}{l}
\bar{C}_{n m} \\
\bar{S}_{n m}
\end{array}\right|=\left|\frac{(n+m) !}{(n-m) !(2 n+1) k}\right|^{1 / 2}\left|\begin{array}{l}
C_{n m} \\
S_{n m}
\end{array}\right|
$$

$\mathrm{K}=1 \mathrm{khi} \mathrm{m}=0, \mathrm{k}=2$ khi $\mathrm{m} \neq 0$.

Theo Kaula (1967) phương sai của hệ số hàm điều hoà cầu có thể được biểu thị bằng công thức:

$$
\delta g_{n}^{2}=\sum_{k=0}^{m}\left(\bar{\delta}_{\mathcal{C}_{n k}}^{2}+\bar{\delta}^{2}\right)
$$

Trên cơ sở đó hàm hiệp phương sai theo Peninhen (1970) có thể viết dưới dạng biểu thức:

$$
C(\psi)=\sum_{n=2}^{\infty} \delta g_{n}^{2} P_{n}(\cos \psi)
$$

Theo R.H Rapp (1998) sai số xác định dị thường trọng lực của mô hình trọng trường toàn cầu có thể biểu diễn bằng biểu thức sau: 


$$
\delta g=\frac{G M}{R^{2}} \sum_{m=0}^{n}(n-1) \sqrt{\delta c_{n, m}^{2}+\delta s_{n, m}^{2}}
$$

Trong đó : $\bar{\delta}_{c_{n, m}}, \bar{\delta}_{S_{n, m}} \quad$ - Phương sai của các hệ số điều hoà cầu có bậc $\mathrm{n}$ và hạng m. Đại lượng $\delta \mathrm{g}$ có thể xác định được cho mỗi mô hình trọng trường trên cơ sở sử dụng các sai số chuẩn $\delta \mathrm{c}_{\mathrm{n}, \mathrm{m}}, \delta \mathrm{s}_{\mathrm{n}, \mathrm{m}}$ hệ số cầu của mỗi mô hình trọng trường và giá trị Hằng số trọng trường Trái đất $\mathrm{GM}$ của mô hình trọng trường đó. Ví dụ của mô hình EGM2008, GM=3.986004418x10 $10^{14} \mathrm{~m}^{3} / \mathrm{s}^{2} \ldots$.

Khi đánh giá dị thường trọng lực được biểu thị dưới dạng hàm điều hoà cầu thường được viết dưới dạng biểu thức sau:

$$
\delta g_{\text {Tot }}^{2}=\delta g_{\text {Com }}^{2}+\delta g_{\text {omi }}^{2}
$$

Trong đó: $\delta$ gTot - Tổng các sai số

§gCom - Sai số đại diện.

¿gOmi - Sai số bỏ qua

Sai số bỏ qua được viết bằng công thức sau : $\delta g_{\text {omi }}^{n_{\max }}=\sqrt{\sum_{n=n+1}^{\infty} \Delta g^{2}}$

Theo W.M Kaula (1966) có thể cho:

Sai số đại diện bằng:

$$
\begin{aligned}
& \left(\delta g_{\text {Com }}^{n_{\max }}\right)^{2}=\delta g_{\text {Tot }}^{2}-\left(\delta g^{n}\right. \\
& \text { số đại diện thực được tính bằng } \\
& \delta g_{\text {Com }}^{\text {Real }}=\sqrt{\delta g_{\text {Com }}^{2}-\delta g_{i}^{2}}
\end{aligned}
$$

Theo B.P Simberev (1975) sai số đại diện thực được tính bằng biểu thức:

Trong đó: $\delta g_{\text {Com }}^{\text {Real }}$ - Sai số đại diện thực.

$$
\delta g_{i} \text { - Sai số đo. }
$$

Theo biểu thức (9) đối với mô hình GOCE (n=300): $\delta$ gOmiss $\leq 0,22 \mathrm{mGal}$

Còn đối với mô hình EGM2008 (n=2159) : $\delta$ gOmiss $\leq 0,030 \mathrm{mGal}$.

Có thể cho: $\delta \mathrm{gTot}=\Delta \mathrm{gT}-\Delta \mathrm{gS}$,

Trong đó: $\Delta \mathrm{gT}, \Delta \mathrm{gS}$ là dị thường trọng lực đo trực tiếp và dị thường trọng lực mô hình vệ tinh.

Nếu bỏ qua sai số $\delta g O m i$, sai số đại diện thực có thể viết:

$$
\delta g_{\text {Com }}^{\text {Real }}=\sqrt{\delta g^{2}-\delta g_{i}^{2}}
$$

Biểu thức (12) cho thấy sai số của mô hình vệ tinh chủ yếu phụ thuộc vào sai số của dị thường mặt đất, trong đó sai số các hệ số cầu bậc thấp là chủ yếu. 


\section{Kết quả thử nghiệm và đánh giá độ chính xác mô hình vệ tinh}

\subsection{Khu vục thử nghiệm và dũ liệu}

Trong khoảng thời gian từ năm 2007- 2009 phân viện thành phố Hồ Chí Minh thuộc Viện Khoa học Đo đạc và Bản đồ đã tiến hành đo trọng lực biển bằng máy trọng lực Dynamic Gravimeter của hãng ZLS (Mỹ). Việc đo trọng lực biển được thực hiện trên một số khu vực chạy dọc theo bờ biển từ Hà Tiên đến Quảng Ninh. Đo trọng lực biển bằng tầu biển có trọng tải khoảng 500 tấn, toạ độ được xác định bằng công nghệ DGPS có sai số định vị với độ chính xác 1-3 $\mathrm{m}$, độ sâu đo với máy hồi âm có độ chính xác $0,01 \%$ h (h-độ sâu của nước biển), Máy đo với độ chính xác $1 \mathrm{mGal}$. Trên cơ sở những tính năng kỹ thuật trên đã tiến hành đo trọng lực và xác định dị thường trọng lực khoảng không tự do và dị thường Bouger trên một số khu vực vùng Biển Việt Nam. Kết quả xử lý đánh giá độ chính xác của giá trị đo trọng lực đạt $\pm 1,3 \mathrm{mGal}$, còn dị thường trọng lực khoảng không tự do cho độ chính xác khoảng $\pm 1,4$ mGal.

\section{Khu vực Biển Vịnh Bắc bộ}

Vị trí khu đo:

Vĩ độ: 18046’27",3 $\leq \varphi \leq 20^{\circ} 12$ '24",0 Kinh độ: $106^{0} 27^{\prime} 18^{\prime \prime}, 0 \leq \lambda \leq 107^{\circ} 45^{\prime} 12^{\prime \prime}, 0$

Diện tích $11165 \mathrm{~km}^{2}$. Khu đo thực hiện tổng cộng 58989 điểm

Khu vực vịnh Bắc Bộ được khảo sát đo trọng lực từ độ sâu 30-100m nước. Khu đo đã được tính dị thường khoảng không tự do và dị thường Bouger. Theo kết quả đánh giá độ chính xác của phân viện Đo đạc và Bản đồ phía nam độ chính xác trị đo đạt $1,29 \mathrm{mGal}$, độ chính xác dị thường khoảng không tự do 1,4 mGal.

\section{Khu vực Biển Hà Tiên - Phú Quốc}

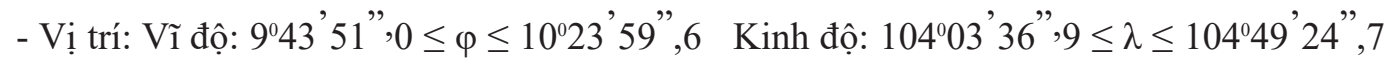

Diện tích khoảng: $\mathrm{S}=2816 \mathrm{~km}^{2}$. Khu đo thực hiện có tổng cộng 138.850 điểm.

Theo kết quả đo đạc và xử lý tính toán và đánh giá độ chính xác các dữ liệu đo trọng lực của phân viện nghiên cứu Đo đạc và Bản đồ phía nam thì độ chính xác của giá trị trọng lực đo được đạt 1.33 $\mathrm{mGal}$ và sai số dị thường khoảng không tự do là $1,4 \mathrm{mGal}$. Sai số trên đã được tính toán kiểm tra bằng sai số lý thuyết và sai số đo kiểm tra trên 357 điểm giao cắt đều phù hợp và tương đương nhau.

\subsection{Mô hình trọng trường toàn cầu}

Theo International Centre for Global Earth Models (ICGEM), Các tổ chức thu thập, xử lý tính toán dữ liệu trọng lực, mặt đất, tàu biển, hàng không và vệ tinh đã tổng hợp và cung cấp 175 mô hình trọng trường toàn cầu, trong đó có 4 mô hình có dữ liệu tương đối đầy đủ, được thành lập trong những năm gần đây bằng các công nghệ hiện đại và có bậc cao nhất hiện nay thỏa mãn việc sử dụng cho các tính toán xử lý ở độ chính xác và mật độ cao như sau: (Xem bảng 1)

Các mô hình được cung cấp chính thức và hợp pháp cho người sử dụng trên toàn cầu dưới dạng các hệ số điều hòa cầu $\mathrm{C}_{\mathrm{lm}}$ và $\mathrm{S}_{\mathrm{lm}}$, chúng ta sẽ thực hiện các phép tính toán để chuyển từ mô hình dạng các hệ số điều hòa cầu thành mô hình Grid dị thường trọng lực với kích thước các ô chuẩn là 3'x3', phạm vi mô hình sẽ tính toàn cho phần lãnh thổ, lãnh hải Việt Nam là:

- Vĩ độ từ $6^{0}$ đến $24^{\circ}$, Kinh độ từ $102^{\circ}$ đến $118^{\circ}$ 
- Tọa độ trong hệ WGS-84- Giá trị dị thường trọng lực khoảng không tự do (Free - Air)

- Mô hình EGM2008, được thành lập và công bố năm 2008 từ các dữ liệu trọng lực Altimetry, trọng lực mặt đất và trọng lực vệ tinh quỹ đạo thấp $\mathrm{S}$ (Grace), với bậc tối đa của các hệ số điều hòa cầu là 2159 và bậc mở rộng tới 2190.

- Mô hình EIGEN-6C4, được thành lập và công bố năm 2014 từ các dữ liệu trọng lực Altimetry, trọng lực mặt đất và trọng lực vệ tinh quỹ đạo thấp $\mathrm{S}($ Goce), $\mathrm{S}$ (Grace), $\mathrm{S}$ (Lageos), với bậc tối đa của các hệ số điều hòa cầu là 2190 .

- Mô hình GECO, được thành lập và công bố năm 2015 từ các dữ liệu trọng lực của mô hình EGM2008 kết hợp với dữ liệu trong lực của vệ tinh S(GOCE) tới bậc trung (n=300), các bậc cao tới n=2190 sử dụng của mô hình EGM2008.

- Mô hình SGG-UGM-1, Là một hình cải tiến của nhóm nhà khoa học Liang, W. et al., và Xu, $\mathrm{X}$. et al. Được thành lập và công bố năm 2018 từ các dữ liệu trọng lực của mô hình EGM2008 kết hợp với dữ liệu trong lực của vệ tinh $\mathrm{S}(\mathrm{GOCE})$, với bậc tối đa của các hệ số điều hòa cầu là 2159 .

Việc thực hiện tính toán kiểm tra 4 mô hình trọng trường toàn cầu ở trên bằng các dữ liệu trọng lực đo tầu biển tại vùng biển ở 2 khu vực vịnh Bắc bộ và Hà Tiên - Phú Quốc.

Bảng 1: Các mô hình trọng trường toàn cầu đura vào tính toán

\begin{tabular}{|c|l|c|c|l|}
\hline Nr & \multicolumn{1}{|c|}{ Model } & Year & Degree & \multicolumn{1}{|c|}{$\begin{array}{c}\text { Data:(A= TL đo cao vệ tinh Altimetry) } \\
(\text { G= TL Mặt Đât)(S= TL Vệ Tinh) }\end{array}$} \\
\hline 152 & GECO & 2015 & 2160 & EGM2008, S(Goce) \\
\hline 148 & EIGEN-6C4 & 2014 & 2190 & A, G, S(Goce), S(Grace), S(Lageos) \\
\hline 104 & EGM2008 & 2008 & 2190 & A, G, S(Grace) \\
\hline 167 & SGG-UGM-1 & 2018 & 2159 & EGM2008, S(Goce) \\
\hline
\end{tabular}

\subsection{Kết quả đánh giá}

\section{Khu vục vùng Biển Vịnh Bắc bộ}

Kết quả đánh giá khu vực vùng Biển Vịnh Bắc bộ được thể hiện ở bảng 3 cho thấy các mô hình trọng trường toàn cầu cho độ chính xác chưa cao, do tiếp giáp với khu vực Hải Phòng - Quảng Ninh có rất nhiều đảo địa hình không bằng phẳng có nhiều đảo có độ cao tới $100 \mathrm{~m}$ (Cô Tô), do vậy ảnh hưởng của địa hình rất đáng kể. Vì vậy các kết quả này chưa phản ánh đúng độ chính xác của các mô hình. Mặt khác theo công bố của NGA (National Geospatial- intelligence Agency) trong phạm vi khu vực nước ta là vùng "Fill-in" tức là chưa có dữ liệu trọng lực mặt đất bổ sung cho trọng lực vệ tinh. Do vậy mô hình trọng trường toàn cầu có độ chính xác chưa cao. Những khu vực biển ở Vịnh bắc bộ thiếu dữ liệu trọng lực đo trực tiếp, cần bổ sung dữ liệu trọng lực vệ tinh cần thiết phảihiệu chỉnh vào dữ liệu trọng lực vệ tinh các số cải chính địa hình phần dư RTM (Residual Terrain Model) sẽ cho độ chính xác tốt hơn tiệm cận với độ chính xác của trọng lực mặt đất. (Xem bảng 2)

Qua các kết quả tính toán và phân tích ở trên cho thấy, dữ liệu dị thường trọng lực khoảng không tự do từ mô hình trọng trường toàn cầu GECO là gần với dữ liệu đo trọng lực bằng tầu biển nhất, biểu đồ phân bố độ lệch chuẩn hội tụ quanh mốc " 0 ” cũng nhiều nhất, sai số trung phương trung bình so với dị thường trọng lực đo từ tầu biển cũng nhỏ nhất. Mô hình EGM2008 và đặc biệt là mô hình EIGEN 6C4 đạt độ chính xác không cao do chưa được tính số cải chính địa hình RTM. Sai số của mô hình GECO ở mức 4 mGal là phù hợp với báo cáo của các tài liệu quốc tế [6] như NGA, 
DTU Space cung cấpvề đánh giá sai số của trọng lực vệ tinh ở khu vực Biển Đông của Việt Nam từ $3 \mathrm{mGal}$ đến $5 \mathrm{mGal}$ như hình 1 sau:

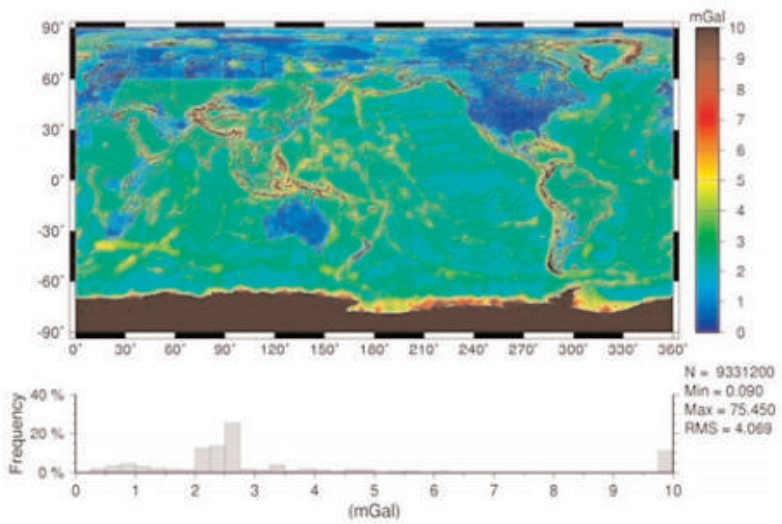

Hình 1: Độ lệch của mô hình trọng trường toàn cầu so với trọng lục mặt đất

Bảng 2: Kết quả đánh giá Mô hình trọng truờng vệ tinh với các dũ liệu trọng lực mặt đất,

$$
\text { Sai số } T P T B=\sqrt{\frac{\sum \delta g^{2}}{n}} \text {, vói } \delta g=\Delta g_{T}-\Delta g_{S}
$$

\begin{tabular}{|l|r|r|r|r|r|}
\hline \multicolumn{1}{|c|}{ Models } & \multicolumn{1}{c|}{$\begin{array}{c}\text { Bậc } \\
(\boldsymbol{n}=\boldsymbol{m})\end{array}$} & $\begin{array}{l}\Delta \boldsymbol{g}_{\min } \\
(\boldsymbol{m} \text { Gal })\end{array}$ & $\begin{array}{l}\Delta \boldsymbol{g}_{\max } \\
(\boldsymbol{m} \text { Gal })\end{array}$ & $\begin{array}{l}\Delta \boldsymbol{g}_{\text {mean }} \\
(\boldsymbol{m} \text { Gal })\end{array}$ & $\begin{array}{l}\text { Sai số } \\
(\boldsymbol{m} \text { Gal })\end{array}$ \\
\hline EGM 2008 & 2159 & -47.219 & 7.443 & -27.967 & $\mathbf{5 . 5 0 3}$ \\
\hline $\begin{array}{l}\text { EIGEN } \\
\text { 6C4 }\end{array}$ & 2190 & -58.957 & 10.647 & -31.126 & $\mathbf{1 1 . 2 2 6}$ \\
\hline $\begin{array}{l}\text { SGG- } \\
\text { UGM-1 }\end{array}$ & 2159 & -47.288 & 4.108 & -28.201 & $\mathbf{4 . 8 7 2}$ \\
\hline GECO & 2190 & -50.251 & 0.543 & -29.409 & $\mathbf{4 . 3 4 1}$ \\
\hline
\end{tabular}

Biểu đồ độ chênh giá trị dị thường khoảng không tự do của các mô hình so với giá trị dị thường khoảng không tự do của tuyến đo trên tầu biển khu phía Bắc như sau: (Trục ngang là số hiệu điểm, trục đứng là giá trị dị thường trọng lụcc):

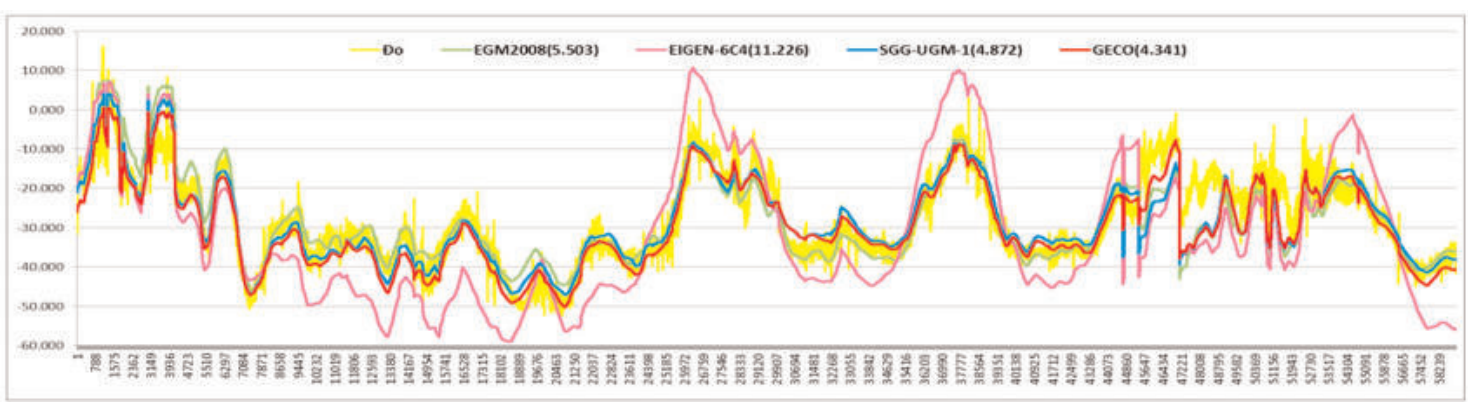

Hình 2: Biểu đồ độ chênh giá trị dị thwờng khoảng không tự do của các mô hình vệ tinh so với đo tầu biển khu vục Vịnh Bắc bộ phía Bắc

Biểu đồ độ lệch chuẩn trọng lực vệ tinh toàn cầu so với dữ liệu đo trực tiếp từ tàu Biển khu vực phía Bắc như sau: 


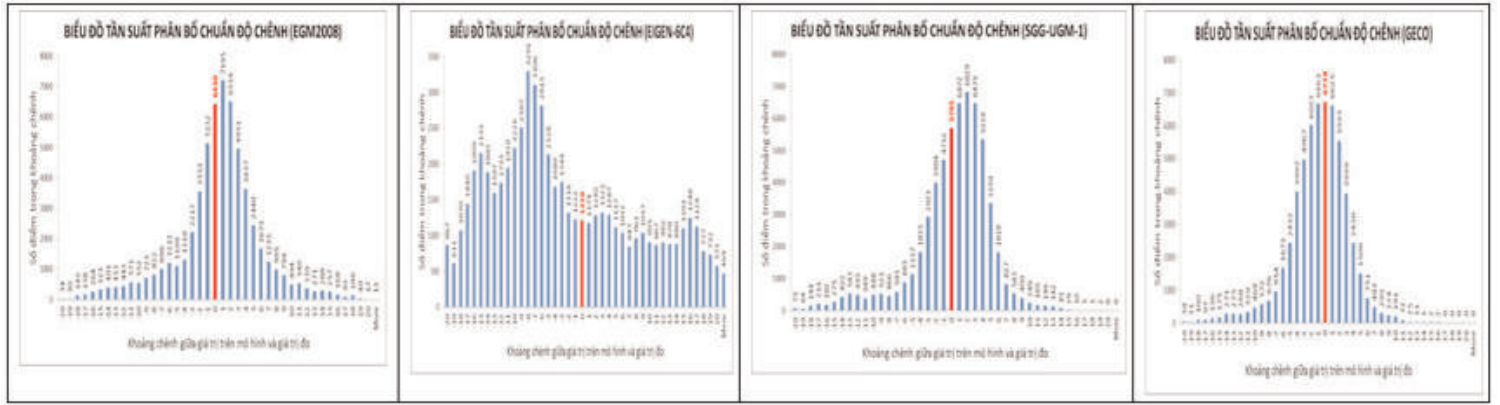

Hình 3: Độ lệch chuẩn của mô hình EGM2008, EIGEN-6C4, SGG-UGM-1

và GECO trên tuyến đo phía Bắc

\section{Khu vực vùng Biển Hà Tiên -Phú Quốc}

Dữ liệu gồm nhiều tuyến đo chạy vuông góc đan xen với nhau từ khu vực bờ biển Kiên Giang tới đảo Phú Quốc, các tuyến đo chạy hình zic zắc liên tục và lấp đầy một vùng biển khép kín, được thực hiện vào giữa tháng 4 năm 2008. Phần lớn các tuyến đo đi vào vùng biển phẳng lặng, quang đãng, không có sự biến đổi nhiều của độ sâu địa hình đáy biển, không có các đảo nhỏ và bãi ngầm, chỉ có một lượng nhỏ điểm đo ở gần bờ và đi cạnh một số đảo gần, toàn bộ các tuyến đo có hình màu xanh như hình dưới đây.

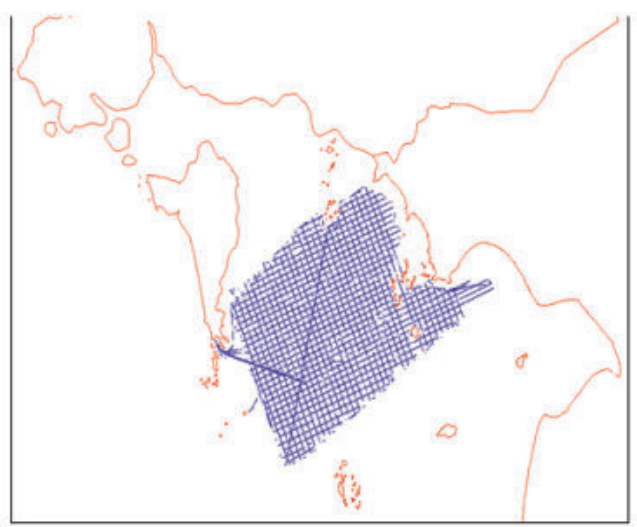

Hình 4: Các tuyến đo trọng lực tầu biển khu vục vùng biển Hà Tiên-Phú Quốc ở phía Nam

Kết quả tính toán sai số trung phương trung bình của dị thường khoảng không tự do từ các mô hình trọng trường toàn cầu so với dữ liệu đo trực tiếp từ tàu Biển như sau:

Bảng 3: Kết quả đánh giá Mô hình trọng trương toàn cầu với các dũ liệu trọng lục mặt đất,

$$
\text { TPTB }=\sqrt{\frac{\sum \delta g^{2}}{n}} \text {, Vóo } i \delta g=\Delta g_{T}-\Delta g_{S}
$$

\begin{tabular}{|c|c|c|c|c|c|}
\hline Models & $\begin{array}{c}\text { Bậc } \\
(n=m)\end{array}$ & $\begin{array}{c}\Delta g_{\min } \\
(m G a l)\end{array}$ & $\begin{array}{c}\Delta g_{\max } \\
(m G a l)\end{array}$ & $\begin{array}{l}\Delta g_{\text {mean }} \\
(m G a l)\end{array}$ & $\begin{array}{l}\text { Sai số } \\
(m G a l)\end{array}$ \\
\hline EGM 2008 & 2159 & -12.770 & 3.890 & -2.994 & 4.928 \\
\hline EIGEN $6 \mathrm{C} 4$ & 2190 & -9.962 & 6.985 & 0.656 & 3.622 \\
\hline SGG-UGM-1 & 2159 & -11.014 & 7.099 & -0.429 & 3.585 \\
\hline GECO & 2190 & -8.456 & 7.962 & 0.406 & 3.098 \\
\hline
\end{tabular}


Biểu đồ độ chênh giá trị dị thường khoảng không tự do của các mô hình so với giá trị dị thường khoảng không tự do của tuyến đo trên tầu biển khu Hà Tiên-Phú Quốc như sau (Trục ngang là số hiệu điểm, trục đứng là giá trị dị thuờng trọng lục)

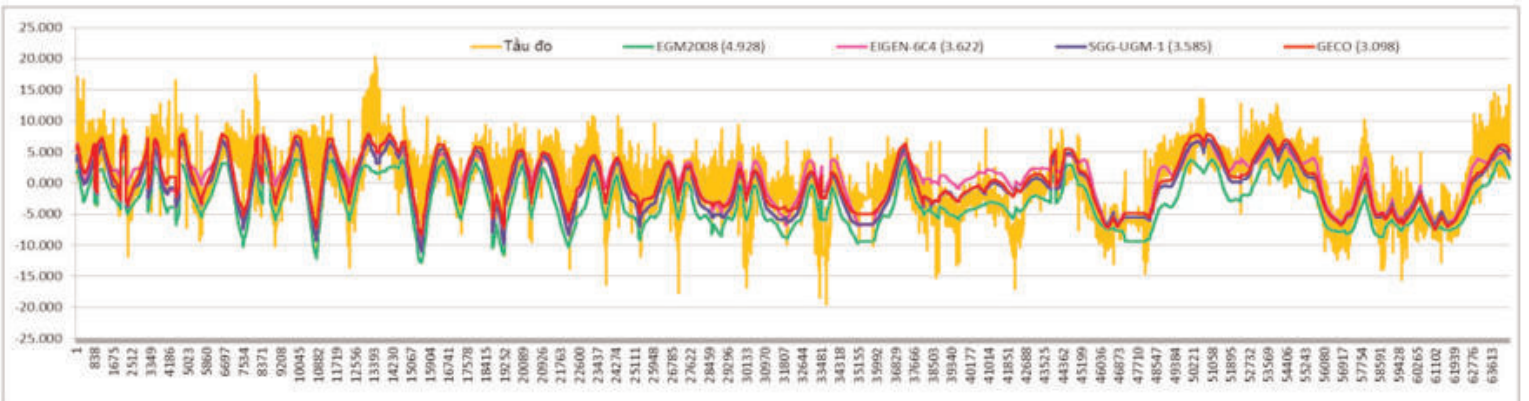

Hình 5: Biểu đồ độ chênh giá trị dị thường của các mô hình so với đo tầu biển khu vực vùng Biển Hà Tiên-Phú Quốc ở phía Nam

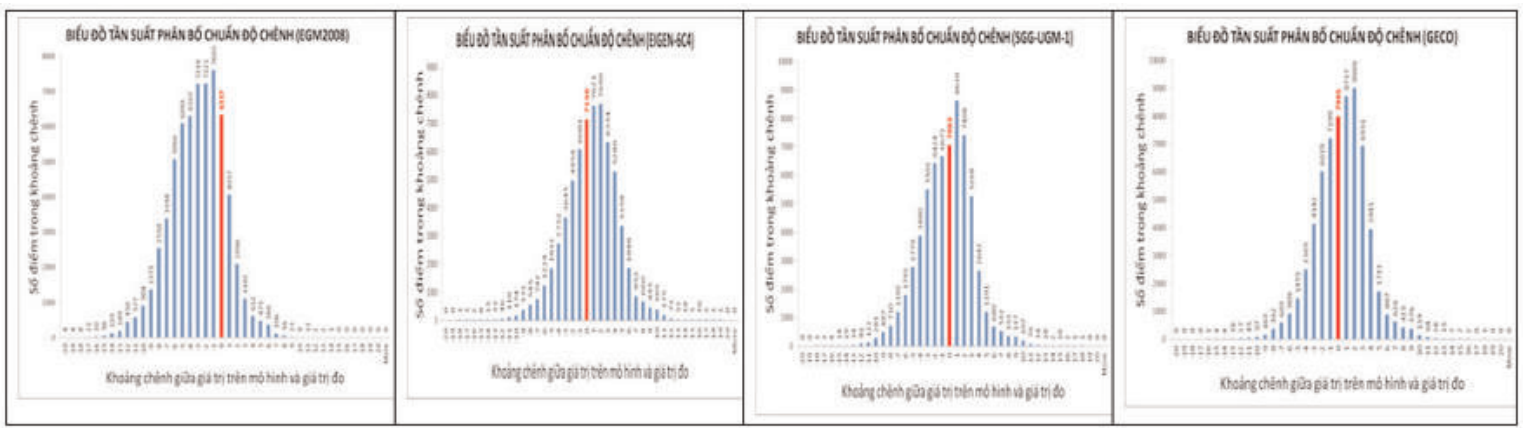

Hình 6: Độ lệch chuẩn của mô hình EGM2008, EIGEN-6C4, SGG-UGM-1 và GECO trên tuyến đo phía Nam

Theo công thức (12), ta có sai số đại diện dị thường trọng lực bằng:

$$
\delta \mathrm{g}^{2} \mathrm{Com}=\left(\delta \mathrm{g}^{2} \mathrm{Tot}^{-} \delta \mathrm{g}_{\mathrm{i}}^{2}\right)
$$

Như vậy với các kết quả đo các khu vực ta có bảng kết quả sai số như sau:

Khu đo vinh Bắc bô

\begin{tabular}{|c|l|c|c|c|}
\hline STT & \multicolumn{1}{|c|}{ Mô hình } & $\begin{array}{c}\text { Sai số tồng } \\
\delta \mathrm{g}_{\text {Tot }}(\mathrm{mGal})\end{array}$ & $\begin{array}{c}\text { Sai số đo } \\
\delta \mathrm{g}_{\mathrm{i}}(\mathrm{mGal})\end{array}$ & $\begin{array}{c}\text { Sai số đại diện } \\
\delta \mathrm{g}_{\mathrm{Com}}(\mathrm{mGal})\end{array}$ \\
\hline 1 & EGM2008 & 5,503 & 1,29 & 5,36 \\
\hline 2 & EIGEN 6C4 & 11,226 & 1,29 & 11,13 \\
\hline 3 & SGG-UGM-1 & 4,872 & 1,29 & 4,59 \\
\hline 4 & GECO & 4,341 & 1,29 & 4,14 \\
\hline
\end{tabular}

Theo công thức (13) cho thấy các mô hình có sự khác nhau về độ chính xác, sự sai lệch lớn chủ yếu do ảnh hưởng của các hệ số điều hòa cầu bậc thấp khi chưa có các dữ liệu mặt đất tham gia vào xác định mô hình vệ tinh ở vùng biển Việt Nam. Mô hình GECO có độ chính xác tốt hơn do có sự tham gia kết hợp tới bậc 359 của mô hình GOCE với mô hình EGM2008 do vậy sai số các hệ điều hoà bậc thấp giảm đi đáng kể so với các mô hình khác. 
Khu đo Hà Tiên - Phú Quốc

\begin{tabular}{|l|l|l|l|l|}
\hline Stt & Mô hình & $\begin{array}{l}\text { Sai số tồng } \\
\delta \mathrm{g}_{\text {Tot }}(\mathrm{mGal})\end{array}$ & $\begin{array}{l}\text { Sai số đo } \\
\delta \mathrm{g}_{\mathrm{i}}(\mathrm{mGal})\end{array}$ & $\begin{array}{l}\text { Sai số đại diện } \\
\delta \mathrm{g}_{\mathrm{Com}}(\mathrm{mGal})\end{array}$ \\
\hline 1 & EGM2008 & 4,928 & 1,33 & 4,74 \\
\hline 2 & EIGEN 6C4 & 3,622 & 1,33 & 3,37 \\
\hline 3 & SGG-UGM-1 & 3,585 & 1,33 & 3,33 \\
\hline 4 & GECO & 3,098 & 1,33 & 2,80 \\
\hline
\end{tabular}

Qua các kết quả tính toán và phân tích ở trên cho thấy, dữ liệu dị thường trọng lực khoảng không tự do từ mô hình trọng trường toàn cầu GECO là gần với dữ liệu đo trọng lực bằng tầu biển nhất, biểu đồ phân bố độ lệch chuẩn hội tụ quanh mốc " 0 ” cũng nhiều nhất, sai số trung phương trung bình so với dị thường trọng lực đo từ tầu biển cũng nhỏ nhất. Trong khi đó mô hình EGM2008 có sự khác biệt và sai số lớn nhất so với dữ liệu tàu biển và các mô hình đã được cải chính bới dữ liệu trọng lực từ vệ tinh GOCE. Khu vực này ít chịu ảnh hưởng của địa hình đáy biển và địa hình xung quanh nên mô hình EIGEN-6C4 cho kết quả tốt hơn EGM2008. Sai số của mô hình GECO ở mức $3 \mathrm{mGal}$ phản ảnh đúng sai số của mô hình dữ liệu trọng lực vệ tinh ở khu vực Biển khơi khi đã được xử lý tính toán, làm trơn từ mọi nguồn dữ liệu trọng lực và được làm khớp với các dữ liệu trọng lực độ chính xác cao từ vệ tinh GOCE.

\section{Thảo luận và kiến nghị}

\subsection{Thảo luận}

Cho đến thời điểm hiện nay, chỉ có 4 mô hình trọng trường toàn cầu EGM2008, EIGEN-6C4, SGG-UGM-1 và GECO như đã kể trên có số bậc cao nhất lên đến 2190 (Độ phân giải cao nhất), được xây dựng dựa trên việc kết hợp đủ các nguồn dữ liệu mặt đất và các dữ liệu vệ tinh trên toàn cầu, đáp ứng được các tính toán trọng lực cục bộ. Các mô hình trọng trường nêu ở trên có độ chính xác tương đối đồng đều, theo các tài liệu quốc tế và đánh giá nêu trên độ chính xác ở khu vực vùng Biển Đông của Việt Nam đạt từ $3 \mathrm{mGal}$ đến $5 \mathrm{mGal}$, trong đó, ở các khu vực ngoài khơi ít đảo độ chính xác cao hơn khu vực gần bờ.

Sai số các mô hình trọng trường ở mức 3-5 mGal thuộc vùng biển Việt Nam chủ yếu từ các nguồn sai số do thiếu các dữ liệu đo trọng lực biển dẫn đến làm tăng sai số xác định các hệ số điều hoà cầu. Để làm giảm các sai số trên cần thiết phải bổ sung các dữ liệu trọng lực đo trực tiếp và tiến hành hiệu chỉnh bổ sung số cải chính địa hình Biển.

Do mô hình EGM2008 chưa được cải chính bằng các dữ liệu trọng lực độ chính xác cao của vệ tinh GOCE và mô hình EIGEN-6C4 chịu ảnh hưởng nhiểu bởi yếu tố địa hình nên có sai số lớn hơn và có sự khác biệt lớn lên đến $12 \mathrm{mGal}$ so với các mô hình còn lại. Các mô hình trọng trường ở các khu vực gần bờ và có nhiều đảo cần được hiệu chỉnh số cải chính địa hình mới nâng cao độ chính xác. 
Mô hình trọng trường toàn cầu GECO được thành lập ở thời điểm mới nhất (năm 2015) ở bậc cao nhất 2190 trên cơ sở xử lý kết hợp giữa dữ liệu trọng lực từ mô hình EGM2008 với độ phân giải cao, với dữ liệu trọng lực độ chính xác cao tới bậc 359 từ vệ tinh GOCE nên cho độ chính xác cao nhất, tính ổn định nhất và gần với dữ liệu trọng lực đo trên tàu biển nhất khi so với các mô hình còn lại. Sai số trung phương trung bình của độ chênh giữa dị thường trọng lực GECO với tàu biển đạt $\pm 4.341 \mathrm{mGal}$ ở khu vực Biển miền Bắc và $\pm 3.098 \mathrm{mGal}$ ở khu vực Biển miền Nam Việt Nam. Kết quả này phù hợp với các đánh giá về độ chính xác dữ liệu trọng lực vệ tinh trên biển của các tổ chức quốc tế như NGA, DTU Space .v.v...

\subsection{Kiến nghị}

Sử dụng mô hình trọng trường toàn cầu GECO thành lập năm 2015 ở bậc cao nhất 2190 và chuyển về ô chuẩn trọng lực 3'x3'để phục vụ cho việc làm khớp (Fitting) mô hình vệ tinh với các dư liệu đo trọng lực ở vùng biển Việt Nam.

Có thể sử dụng dữ liệu trọng lực từ mô hình trọng trường toàn cầu độ phân giải cao để xây dựng CSDL trọng lực phủ kín toàn bộ vùng biển Việt Nam với độ chính xác đồng nhất phù hợp với các khu vực ngoài khơi xa bờ, ít chịu ảnh hưởng của địa hình làm cơ sở để thiết kế tổng thể số lượng, mật độ, vị trí các điểm trọng lực đo trực tiếp để xử lý làm khớp với dữ liệu trọng lực vệ tinh sẽ tiết kiệm được các số lượng điểm đo mặt đất.

Sử dụng các dữ liệu trọng lực đo bằng tầu biển, hàng không trên biển có độ chính xác, độ tin cậy cao tại các khu vực đặc trưng phân bố trên vùng biển Việt Nam để cải chính lại các dữ liệu trọng lực vệ tinh cho phù hợp với dữ liệu cục bộ.

Sử dụng dữ liệu trọng lực vệ tinh từ mô hình trọng trường toàn cầu GECO đã được cải chính với dữ liệu trọng lực đo trực tiếp để bổ sung, lấp kín các khu vực khó tiếp cận và các khu vực chồng lắp thuộc vùng đặc quyền kinh tế trên Biển nhằm đảm bảo xây dựng được một CSDL trường trọng lực độ chính xác cao phủ kín, liên tục toàn bộ khu vực Biển Đông của Việt Nam và lân cận, phục vụ các nhu cầu thăm dò khoáng sản, dự báo thiên tai và phát triển kinh tế, an ninh, quốc phòng cũng như sự toàn vẹn lãnh hải, lãnh thổ Việt Nam. $\bigcirc$

\section{Tài liệu tham khảo}

[1]. Franz Barthelmes. Definition of Functionals of the Geopotential and Their Calculation from Spherical Harmonic Models, Scientific Technical Report, Potsdam, Germany January 2013, ISSN 1610-0956, DOI: 10.2312/GFZ.b103-0902-26.

[2]. Förste, Christoph et al, 2014 Förste, Christoph, Bruinsma, Sean L., Abrikosov, Oleg, Lemoine, Jean-Michel, Marty, Jean Charles, Flechtner, Frank, Balmino, G., Barthelmes, F., Biancale, R.; EIGEN-6C4 The latest combined global gravity field model including GOCE data up to degree and order 2190 of GFZ Potsdam and GRGS Toulouse; GFZ Data Services, doi: 10.5880/ICGEM.2015.1, 2014.

[3]. Gilardoni, M. et al, 2016, Gilardoni, M., Reguzzoni, M., Sampietro, D.; GECO: A global gravity model by locally combining GOCE data and EGM2008; Studia Geophysica et Geodaetica, 
Vol 60, p. 228-247, doi: 10.1007/s11200-015-1114-4, 2016.

[4]. Liang, W. et al., 2018 \& Xu, X. et al. (2017) Liang W., Xu X., Li J., Zhu G., 2018: The determination of an ultra high gravity field model SGG-UGM-1 by combining EGM2008 gravity anomaly and GOCE observation data Acta Geodaeticaet Cartographica Sinica, 47(4): 425-434. DOI:10.11947/j.AGCS.2018.20170269.

[5]. Pavlis, N.K. et al, 2008. Pavlis, N.K., Holmes, S.A., Kenyon, S.C., Factor, J.K.;An Earth Gravitational Model to Degree 2160: EGM2008; Vienna, Austria, 2008.

[6]. Nguyễn Tuấn Anh, 2018. Nghiên cứu phương pháp hiệu chỉnh các hệ số điều hòa cầu của mô hình trọng trường Trái đất EGM2008 bằng dữ liệu trọng lực ở Việt Nam.

[7]. Đặng Hùng Võ, Lê Minh, nnk 2005. Xây dựng CSDL trường trọng lực toàn cầu,thiết lập mô hình Geoid độ chính xác cao trên lãnh thổ Việt Nam phục vụ nghiên cứu hoạt động của trái đất và đổi mới công nghệ đo cao bằng hệ thống định vị toàn cầu, Báo cáo tổng kết Khoa học và kỹ thuật. Hà Nội tr174.

[8]. Trần Đình Âu. Nghiên cứu ứng dụng đo trọng lực Biển ở Việt Nam (Đề tài nghiên cứu cấp Bộ-2006).

[9]. Phân viện Đo đạc và Bản đồ phía nam (Thành phố Hồ chí Minh), Dữ liệu dự án 47 (20072009). $\mathrm{O}$

\section{Summary}

\section{Evaluation global Geopotential Models using Terrectrial Gravity on the Sea of Vietnam}

Le Minh, Vietnam Association of Geodesy Cartography and Remote Sensing

Nguyen Tuan Anh, Department of Survey, Mapping and Geographic information Vietnam

Using Global Gravity Models Data is most important method for establised unified gravity data base covering hold country of Vietnam. For this job need determination of the GGMs with high accuracy and better resolution of the gravity anormalies for combinated with terrectrial gravity observation on the sea of Vietnam. Gravity anormalies from Four selected GGMs: EGM2008 $(n=2159)$, GECO ( $n=2190)$, EIGEN 6C4 (2190) and SGG-UGM-1 are using for analized andcompared with gravity anomalies derived from terrectrial gravity observation on the two site of the sea Vietnam. The Comparison and the results of analized gravity anomalies between GGMs and terrectrial gravity was shown Global gravity model GECO is most accurated model with residual 4,3 mGal for the northern site and 3,0 for Southern site of sea Vietnam. From the results show that global gravity model GECO with the spherical hamonic coefficients up to 2190 is the most suitable model can be use for combination with terrectrial gravity for establised unified gravity data base corvering over sea of Vietnam. $O$ 